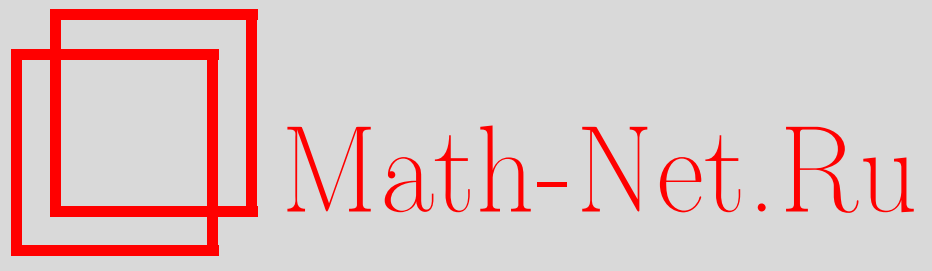

Б. П. Харламов, Плотность распределения точки первого выхода диффузионного процесса из малой окрестности его начальной точки, Теория вероятн. и ее примен., 2000, том 45, выпуск $3,536-554$

DOI: https://doi.org/10.4213/tvp484

Использование Общероссийского математического портала Math-Net.Ru подразумевает, что вы прочитали и согласны с пользовательским соглашением http://www . mathnet.ru/rus/agreement

Параметры загрузки:

IP : 54.157 .27 .8

26 апреля 2023 г., 18:08:12

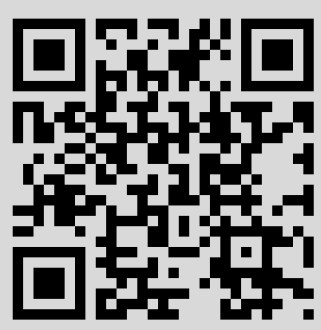




\section{ПЛОТНОСТЬ РАСПРЕДЕЛЕНИЯ ТОЧКИ ПЕРВОГО ВЫХОДА ДИФФУЗИОННОГО ПРОЩЕССА ИЗ МАЛОЙ ОКРЕСТНОСТИ ЕГО НАЧАЛЬНОЙ ТОЧКИ ${ }^{1)}$}

Рассматривается диффузионный процесс в $d$-мерном пространстве. Исследуется распределение точки первого выхода процесса из малой окрестности его начальной точки. Получено слабое асимптотическое разложение плотности этого распределения по малому параметру масштаба. В случае сферических окрестностей выведены простые формулы для первых трех коэффициентов разложения, отражающих вероятностный смысл коэффициентов частного дифференциального уравнения эллиптического типа.

Ключевые слова и фразы: диффузионный процесс, момент первого выхода, точка первого выхода, частное дифференциальное уравнение, эллиптический тип, функция Грина, интегральное уравнение, слабая асимптотика плотности.

\section{1. Введение, определения и обозначения}

В настоящей статье мы рассматриваем полумарковские процессы диффузионного типа в области $S \subset \mathbf{R}^{d}(d \geqslant 2)$. Так мы будем называть непрерывные полумарковские процессы [5], [6], переходные функции которых порождаются в этой области частным дифференциальным уравнением второго порядка эллиптического типа. Основная идея настоящего исследования состоит в нахождении для данных процессов локальной характеризации наподобие условий Колмогорова, которые позволяют для марковского диффузионного процесса в терминах распределений значений процесса в момент времени, стремящийся к нулю, вывести дифференциальные уравнения параболического типа - прямое и обратное уравнения Колмогорова, которым удовлетворяют переходные плотности процесса (см. $[1$, с. 221]). К сожалению, для полумарковских процессов обшего вида, которыми мы занимаемся, распределения в фиксированный (неслучайный) момент времени бесполезны, так как такие

\footnotetext{
* Институт проблем машиноведения РАН, Большой пр., 61, ВО, 199178 С.-Петербург, Россия.

1) Работа выполнена при финансовой поддержке Российского фонда фундаментальных исследований, грант №95-01-01440а.
} 
процессы не обладают, вообще говоря, марковским свойством относительно этих моментов времени. Для полумарковских процессов остается в силе другой вид локальной характеризации - по их распределению первого выхода из малой окрестности начальной точки.

Сравнительно просто решается вопрос о локальной характеризации в терминах первого выхода для одномерных полумарковских процессов диффузионного типа (см. [6]). Это связано с исключительной простотой распределения первого выхода из окрестности начальной точки, которое сосредоточено в двух точках - границах отрезка. Однако и здесь вывести из свойств дифференциального уравнения, которому удовлетворяют переходные функции процесса, локальные свойства распределений первого выхода (обратная задача) есть задача значительно более сложная, чем, имея локальные свойства этих распределений, вывести соответствующее дифференциальное уравнение (прямая задача).

В настояшей работе мы решаем обратную задачу для многомерного полумарковского процесса диффузионного типа: по данному дифференциальному уравнению, которому удовлетворяют переходные производящие функции процесса, найти асимптотические локальные свойства распределений точки первого выхода из малой окрестности начальной точки процесса. Мы надеемся, что полученные на этом пути результаты будут интересны и для специалистов по марковским диффузионным процессам, также являюшимся представителями (может быть - наиболее важными) класса диффузионных полумарковских процессов.

1.1. Непрерывные полумарковские процессы. Исходным объектом для нас является пространство $\mathscr{C}$ всех непрерывных функций $\xi: \mathbf{R}_{+} \rightarrow \mathbf{R}^{d}(d \geqslant 2)$, а также $\sigma$-алгебра $\mathscr{F}$ его борелевских подмножеств. Как правило, $(\mathscr{C}, \mathscr{F})$ играет у нас роль вероятностного пространства. В тех немногих случаях, когда нам потребуется ввести добавочную «случайность», мы будем расширять это пространство по схеме произведения. Пусть $\left(\mathscr{F}_{t}\right)_{t \geqslant 0}$ - натуральная фильтрация, т.е. возрастающий поток $\sigma$-алгебр, порожденных течением процесса на интервале $[0, t]: \mathscr{F}_{t}=\sigma\left(\pi_{s}, s \leqslant t\right)$, где $\pi_{s}: \mathscr{C} \rightarrow \mathbf{R}^{d}$ - однокоординатная проекция $\left(\pi_{s}(\xi)=\xi(s)\right)$, и $\mathscr{T}-$ множество моментов остановки относительно этой фильтрации. В основном, мы будем иметь дело с моментами первого выхода $\sigma_{G} \in \mathscr{T}$, где $G \in \mathfrak{A}$ и $\mathfrak{A}-$ множество всех открытых подмножеств пространства $\mathbf{R}^{d}$.

Рассмотрим $\left(\mathbf{P}_{p}\right)_{p \in \mathbf{R}^{d}}$ - измеримое семейство вероятностных мер на $\mathscr{F}$, для которых $\mathbf{P}_{p}\left\{\pi_{0}=p\right\}=1$. Семейство мер $\left(\mathbf{P}_{p}\right)$ называется полумарковским, если оно обладает марковским (однородным) свойством относительно любого $\sigma_{G}$ (см. [1]). Под непрерывным полумарковским процессом мы понимаем измеримое пространство $(\mathscr{C}, F)$ с заданным на нем полумарковским семейством вероятностных мер. Согласно определению, любой непрерывный строго марковский процесс является не- 
прерывным полумарковским процессом. В частности, таковым будет любой марковский диффузионный процесс. Все полученные в данной статье результаты относятся и к этим процессам, хотя при выводе наших результатов мы не используем марковость процесса.

Полумарковское семейство мер полностью определяется набором переходных функций полумарковского процесса $\left(F_{G}\right)_{G \in \mathfrak{x}}$ :

$$
F_{G}(A \times B \mid p)=\mathbf{P}_{p}\left\{\sigma_{G} \in A, \pi_{G} \in B\right\}, \quad A \in \mathscr{B}\left(\mathbf{R}_{+}\right), B \in \mathscr{B}\left(\mathbf{R}^{d}\right)
$$

$(\mathscr{B}$ - борелевские $\sigma$-алгебры подмножеств соответствующих пространств), где при условии $\sigma_{G}<\infty$ принято обозначение $\pi_{G}(\xi)=\pi_{s}(\xi)$, $s=\sigma_{G}(\xi)$. Мы называем $\pi_{G}(\xi)$ точкой первого выхода реализации процесса $\xi$ из множества $G$; по определению, $\pi_{G}(\xi)=\xi(0)$, если $\xi(0) \notin G$. Набор переходных функций полумарковского процесса согласован специальным условием, которое является следствием марковского свойства относительно любого момента первого выхода из открытого множества. Это условие удобно формулировать в терминах преобразования Лапласа по первой компоненте от переходной функции. Пусть

$$
f_{G}(\lambda, B \mid p)=\int_{0}^{\infty} e^{-\lambda t} F_{G}(d t \times B \mid p) \quad(\lambda \geqslant 0) .
$$

Мы будем называть $f_{G}$ переходной производящей функцией полумарковского процесса. Условие согласования состоит в следующем: для любых $G_{1}, G_{2} \in \mathfrak{A}$ таких, что $G_{1} \subset G_{2}$, справедливо

$$
f_{G_{2}}(\lambda, B \mid p)=\int_{\mathbf{R}^{d}} f_{G_{2}}\left(\lambda, B \mid p_{1}\right) f_{G_{1}}\left(\lambda, d p_{1} \mid p\right) .
$$

Согласно определению семейства $\left(f_{G}\right)$, для $p \in G$ имеем $f_{G}(\lambda, B \mid p)=$ $f_{G}(\lambda, B \cap \partial G \mid p)$, где $\partial G$ - граница $G$, а для $p \notin G$ из очевидного

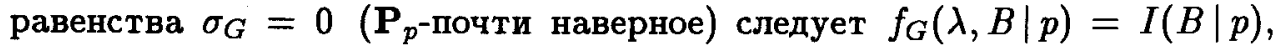
где $I(B \mid \cdot)$ - индикатор множества $B$. При любом $\lambda \geqslant 0$ ядру $f_{G}(\lambda, B \mid p)$ мы сопоставим оператор, отображающий множество интегрируемых функций в себя, обозначенный той же буквой: $f_{G}(\lambda, \varphi \mid p)=$ $\int_{\mathbf{R}^{d}} \varphi\left(p_{1}\right) f_{G}\left(\lambda, d p_{1} \mid p\right)$, где $\varphi-$ интегрируемая функция. В терминах этих операторов уравнение (1) может быть переписано в виде:

$$
f_{G_{2}}(\lambda, \varphi \mid p)=f_{G_{1}}\left(\lambda, f_{G_{2}}(\lambda, \varphi \mid \cdot) \mid p\right) .
$$

По существу, это уравнение отражает полугрупповые свойства семейства операторов $\left(f_{G}\right)$. Мы встретимся с ним при анализе семейства решений задачи Дирихле, связанного с уравнением в частных производных эллиптического типа. 


\section{2. Частное дифференциальное уравнение эллиптического} типа. Рассмотрим в области $S \in \mathfrak{A}$ частное дифференциальное уравнение эллиптического типа

$$
L u \equiv \sum_{i, j=1}^{d} a^{i j} u_{i j}^{\prime \prime}+\sum_{i=1}^{d} b^{i} u_{i}^{\prime}-c u=0 .
$$

Здесь $a^{i j}, b^{i}, c$ - непрерывные вещественные функции, заданные на $S$, при любом $p \in S\left(a^{i j}(p)\right)$ - положительно определенная матрица, $c \geqslant 0$, $u_{i j}^{\prime \prime}, u_{i}^{\prime}$ - частные производные второго и первого порядка по $i$ - и $j$-й координатам соответственно, $u$ - неизвестная функция, для которой сушествуют эти производные. Пусть $G \subset S$ и $u_{G}(\varphi \mid \cdot)$ - решение задачи Дирихле для этого уравнения в ограниченной области $G$ с заданной на границе области непрерывной функцией $\varphi$. При сделанных предположениях это решение непрерывно на $G \cup \partial G$. Если функция $\varphi$ определена и непрерывна на всем множестве $S \cup \partial S$, то при нахождении $u_{G}(\varphi \mid \cdot)$ мы берем ее сужение на границу множества $G$. Если при этом доопределить последнюю функцию на всем $S \backslash G$ равенством $u_{G}(\varphi \mid p)=\varphi(p)$, то уравнение (2) для семейства таких операторов будет справедливо при всех $p \in S$. Иными словами, $u_{G}$ представляет собой оператор, отображающий множество непрерывных и ограниченных функций в себя. В дальнейшем мы понимаем решение задачи Дирихле в этом расширенном смысле.

Покажем, что для семейства операторов $\left(u_{G}\right)_{G \in \Re n S}$ также справедливо (2).

Пусть $G_{1}, G_{2} \in \mathfrak{A}, G_{1} \subset G_{2}$ и $p \in G_{1} ; \varphi$ задана на границе $G_{2}$. При этом $u_{G_{2}}(\varphi \mid \cdot)$ и $u_{G_{1}}\left(u_{G_{2}}(\varphi \mid \cdot) \mid \cdot\right)$ удовлетворяют внутри $G_{1}$ уравнению (3). Кроме того, они совпадают на границе этого множества. По теореме единственности решения задачи Дирихле в области $G_{1}$, которая справедлива при наших предположениях о коэффициентах уравнения, получаем $u_{G_{2}}(\varphi \mid \cdot)=u_{G_{1}}\left(u_{G_{2}}(\varphi \mid \cdot) \mid \cdot\right)$ всюду внутри $G_{1}$. Равенство этих функций вне $G_{1}$ вытекает из правила доопределения решения задачи Дирихле.

О п р е д е л е н и е. Непрерывный полумарковский процесс называется полумарковским прочессом диффузионного типа в области $S$, если для любого $\lambda \geqslant 0$ существует дифференциальный оператор эллиптического типа $L=L(\lambda)$ с описанными выше коэффициентами (см. уравнение (3)), так что семейство переходных операторов $\left(f_{G}(\lambda, \cdot \mid \cdot)\right)$ этого процесса совпадает с семейством $\left(u_{G}\right)$ решений задачи Дирихле уравнения $L u=0$.

В дальнейшем будет понятно, что дифференциальный оператор $L(\lambda)$, определяюший переходные функции диффузионного полумарковского процесса, зависит от $\lambda$ только через коэффициент $c=c(\lambda, \cdot)$. 
Известно (см., например, [1]), что оператор задачи Дирихле может быть представлен как интегральный оператор с некоторым ядром $H_{G}(p, B)$, которое для области $G$ с кусочно гладкой границей определяется плотностью $h_{G}\left(p, p_{1}\right)$ относительно лебеговой меры на границе:

$$
H_{G}(p, B)=\int_{B \cap \partial G} h_{G}\left(p, p_{1}\right) d S\left(p_{1}\right) \quad(p \in G) .
$$

Уравнение (2) в этом случае переходит в интегральное уравнение

$$
h_{G_{2}}\left(p, p_{2}\right)=\int_{\partial G_{1}} h_{G_{1}}\left(p, p_{1}\right) h_{G_{2}}\left(p_{1}, p_{2}\right) d S\left(p_{1}\right) .
$$

Для полумарковского процесса с начальной точкой $p \in G$ ядро $h_{G}(p, \cdot) \equiv$ $f_{G}^{\prime}(\lambda, \cdot \mid p)$ интерпретируется как плотность переходной производящей функции полумарковского процесса, т.е. плотность распределения точки первого выхода из области $G$, полученного в результате преобразования Лапласа по аргументу $t \in \mathbf{R}_{+}$из полумарковской переходной функции.

Заметим, что результат преобразования Лапласа по аргументу $t \mathrm{c}$ параметром $\lambda$ эквивалентен результату преобразования самого полумарковского процесса, состоящего в остановке процесса в случайный момент времени $\tau$. Этот случайный момент времени независим от процесса и экспоненциально распределен с тем же параметром $\lambda$ (см. [7]). Именно здесь нам потребовалось бы расширить наше вероятностное пространство.

Нас будет интересовать асимптотика этой плотности при $G \downarrow p$. Мы сохраним за ней обозначение $h$, опуская до времени параметр $\lambda$.

\section{2. Основные результаты}

Рассмотрим уравнение (3) в области $S$. Пусть эта область содержит начало координат - точку 0 . Без потери общности мы можем предположить, что $(\forall i, j) a^{i j}(0)=\delta^{i j}$. Рассмотрим окрестность нуля ограниченную область $G \subset S$ с кусочно гладкой границей, на которой определена плотность $h_{G}\left(p_{0}, \cdot\right)$ распределения точки первого выхода соответствующего полумарковского диффузионного процесса с начальной точкой $p_{0} \in G$. Пусть

$$
R G=\left\{p:\left(\exists p_{1} \in G\right) p=R p_{1}\right\} \quad(R>0) .
$$

При сделанных предположениях будут определены также плотности $h_{R G}\left(p_{0}, \cdot\right)\left(p_{0} \in R G\right)$.

Пусть $Q\left(p_{1}, p_{2}\right)\left(p_{1}, p_{2} \in G\right)$ - функция Грина для оператора Лапласа в области $G$ и $K\left(p_{1}, p_{2}\right)=\partial Q\left(p_{1}, p_{2}\right) / \partial n_{2}$ - производная от функции Грина как функции от второго аргумента по направлению внутренней 
нормали к границе области. С учетом обозначения $p=\left(p^{1}, \ldots, p^{d}\right) \in \mathbf{R}^{d}$ будем обозначать $D_{i} u=\partial u / \partial p^{i}, D_{i j} u=\partial^{2} u / \partial p^{i} \partial p^{j}$. Кроме того, в приведенных ниже интегралах мы обозначаем переменную интегрирования через $p_{1}, p_{2}, \ldots$ и т.д. В этих случаях $D_{i}^{(k)}, D_{i j}^{(k)}, \ldots$ означает, что дифференцирование ведется по координатам точки $p_{k}$. Чтобы избежать большого количества индексов, мы будем применять также другое обозначение для первых координат точки $p=(x, y, z, \ldots)$, а входящие под знак интеграла переменные $x_{k}, y_{k}, z_{k}, \ldots$, означают соответствующие координаты точки $p_{k}$ - переменной интегрирования. В общем случае $a_{m}^{i j}, a_{m n}^{i j}, b_{m}^{i}, \ldots$ означают частные производные от функций $a^{i j}, a^{i j}, b^{i}, \ldots$ по $m$-и $n$-й координатам соответственно. Мы будем обозначать также $\pi_{i}\left(p_{k}\right)=p_{k}^{i}-i$-я координата точки $p_{k}$.

Теорема 1. Пусть коэффичиенты $b^{i}$ непрерывно дифференцируемы в окрестности нуля и $a^{i j}$ дважды непрерывно дифференчируемы в окрестности нуля. Тогда при $R \rightarrow 0$ имеет место слабая асимптоmuxa

$$
\begin{aligned}
& R^{d-1} h_{R G}\left(R p_{0}, R p\right) \\
& =K\left(p_{0}, p\right)+R\left(\sum_{i} b^{i}(0) \int_{G} Q\left(p_{0}, p_{1}\right) D_{i}^{(1)} K\left(p_{1}, p\right) d V\left(p_{1}\right)+X_{1}\left(p_{0}, p\right)\right) \\
& \quad-R^{2}\left(c(0) \int_{G} Q\left(p_{0}, p_{1}\right) K\left(p_{1}, p\right) d V\left(p_{1}\right)+X_{2}\left(p_{0}, p\right)\right)+o\left(R^{2}\right),
\end{aligned}
$$

где Функиия $X_{1}$ ортогональна любой постоянной и любой линейной Фунжиии на граниче, функчия $X_{2}$ ортогональна любой постоянной функции на граниче. ПІри этом

$$
\begin{aligned}
& X_{1}\left(p_{0}, p\right)=\sum_{i j} \sum_{m} a_{m}^{i j}(0) \int_{G} Q\left(p_{0}, p_{1}\right) \pi_{m}\left(p_{1}\right) D_{i j}^{(1)} K\left(p_{1}, p\right) d V\left(p_{1}\right), \\
& X_{2}\left(p_{0}, p\right)=\sum_{i=1}^{6} X_{2 i}\left(p_{0}, p\right)
\end{aligned}
$$

$2 \partial e$

$$
\begin{aligned}
X_{21}\left(p_{0}, p\right)= & \frac{1}{2} \sum_{i j} \sum_{m n} a_{m n}^{i j}(0) \int_{G} Q\left(p_{0}, p_{1}\right) \pi_{m}\left(p_{1}\right) \pi_{n}\left(p_{1}\right) D_{i j}^{(1)} K\left(p_{1}, p\right) d V\left(p_{1}\right), \\
X_{22}\left(p_{0}, p\right)= & \sum_{i} \sum_{m} b_{m}^{i}(0) \int_{G} Q\left(p_{0}, p_{1}\right) \pi_{m}\left(p_{1}\right) D_{i}^{(1)} K\left(p_{1}, p\right) d V\left(p_{1}\right) \\
X_{23}\left(p_{0}, p\right)= & \sum_{i j} \sum_{m} a_{m}^{i j}(0) \sum_{k l} \sum_{n} a_{n}^{k l}(0) \int_{G} Q\left(p_{0}, p_{1}\right) \pi_{m}\left(p_{1}\right) \\
& \times D_{i j}^{(1)} \int_{G} Q\left(p_{1}, p_{2}\right) \pi_{n}\left(p_{2}\right) D_{k l}^{(2)} K\left(p_{2}, p\right) d V\left(p_{2}\right) d V\left(p_{1}\right)
\end{aligned}
$$




$$
\begin{aligned}
X_{24}\left(p_{0}, p\right)= & \sum_{i} b^{i}(0) \sum_{k l} \sum_{n} a_{n}^{k l}(0) \int_{G} Q\left(p_{0}, p_{1}\right) \\
& \times D_{i}^{(1)} \int_{G} Q\left(p_{1}, p_{2}\right) \pi_{n}\left(p_{2}\right) D_{k l}^{(2)} K\left(p_{2}, p\right) d V\left(p_{2}\right) d V\left(p_{1}\right), \\
X_{25}\left(p_{0}, p\right)= & \sum_{i j} \sum_{m} a_{m}^{i j}(0) \sum_{k} b^{k}(0) \int_{G} Q\left(p_{0}, p_{1}\right) \pi_{m}\left(p_{1}\right) \\
& \times D_{i j}^{(1)} \int_{G} Q\left(p_{1}, p_{2}\right) D_{k}^{(2)} K\left(p_{2}, p\right) d V\left(p_{2}\right) d V\left(p_{1}\right), \\
X_{26}\left(p_{0}, p\right)= & \sum_{i} b^{i}(0) \sum_{k} b^{k}(0) \int_{G} Q\left(p_{0}, p_{1}\right) \\
& \times D_{i}^{(1)} \int_{G} Q\left(p_{1}, p_{2}\right) D_{k}^{(2)} K\left(p_{2}, p\right) d V\left(p_{2}\right) d V\left(p_{1}\right) .
\end{aligned}
$$

Приведенные выше коэффициенты разложения плотности распределения точки первого выхода получагот конкретное значение при известном аналитическом выражении ядер $Q\left(p_{1}, p_{2}\right), K\left(p_{1}, p_{2}\right)$. Пусть $G=B \equiv$ $\{p:|p|<1\}$ - единичный шар.

Теорема 2. При тех же условиях на коэффичиенты уравнения (3), что и в теореме 1, при $R \rightarrow 0$ справедливо слабое асимптотическое разложение плотности перөого выхода из малой сферической окрестности:

$$
\begin{aligned}
R^{d-1} h_{R B}(0, R p)= & \frac{1}{\omega_{d}}+R\left(\frac{1}{2 \omega_{d}} \sum_{i=1}^{d} b^{i}(0) \pi_{i}(p)+X_{1}(0, p)\right) \\
& -R^{2}\left(c(0) \frac{1}{2 d \omega_{d}}+X_{2}(0, p)\right)+o\left(R^{2}\right)
\end{aligned}
$$

где функчия $X_{1}$ ортогональна любой постоянной и любой линейной Функчии на граниче, Функчия $X_{2}$ ортогональна любой постоянной бункции на граниче, $\omega_{d}=2 \pi^{(d / 2)} / \Gamma(d / 2)$ - площадь поверхности единичного шара в d-мерном пространстве. При этом для однородного в пространстве прочесса $X_{1}=0 u$

$$
X_{2}(0, p)= \begin{cases}\frac{1}{8(d-2) \omega_{d}}\left(\sum_{i, j=1}^{d} b^{i} b^{j} \pi_{i}(p) \pi_{j}(p)-\frac{1}{d} \sum_{i=1}^{d}\left(b^{i}\right)^{2}\right), & d \geqslant 3, \\ \frac{1}{8 \cdot 2 \pi}\left(\sum_{i, j=1}^{2} b^{i} b^{j} \pi_{i}(p) \pi_{j}(p)-\frac{1}{2} \sum_{i=1}^{2}\left(b^{i}\right)^{2}\right), & d=2 .\end{cases}
$$

\section{3. Доказательство основных результатов}

3.1. Доказательство теоремы 1. Требуется доказать, что для любой непрерывной функции $\varphi(0 \leqslant \varphi \leqslant 1)$, заданной на границе облас- 
ти $G$, интеграл

$$
\begin{aligned}
\int_{\partial G} & \left(R^{d-1} h_{R G}\left(R p_{0}, R p\right)-K\left(p_{0}, p\right)\right. \\
& -R\left(\sum_{i} b^{i}(0) \int_{G} Q\left(p_{0}, p_{1}\right) D_{i}^{(1)} K\left(p_{1}, p\right) d V\left(p_{1}\right)+X_{1}(p)\right) \\
& \left.+R^{2}\left(c(0) \int_{G} Q\left(p_{0}, p_{1}\right) K\left(p_{1}, p\right) d V\left(p_{1}\right)+X_{2}(p)\right)\right) \varphi(p) d S(p)
\end{aligned}
$$

имеет порядок $o\left(R^{2}\right)$ при $R \rightarrow 0$ равномерно по всем таким $\varphi$.

На границе области $R G$ мы рассмотрим функцию $\varphi_{R}$, где $\varphi_{R}(R p)=$ $\varphi(p)(p \in \partial G)$. Интеграл

$$
\begin{aligned}
u_{R G}(\cdot) & \equiv \int_{\partial R G} \varphi_{R}(p) h_{R G}(\cdot, p) d S_{R}(p) \\
& =\int_{\partial G} R^{d-1} h_{R G}(\cdot, R p) \varphi(p) d S(p)
\end{aligned}
$$

по определению является решением задачи Дирихле для уравнения (3) в области $R G$ со значением $\varphi_{R}$ на границе области.

Уравнение $L u=0$ перепишем в виде $\Delta u=-(L-\Delta) u$. Будем для краткости опускать аргумент - функцию $\varphi$ в обозначении решения задачи Дирихле: $u_{G}(p)$ вместо полного обозначения $u_{G}(\varphi \mid p)$. Тогда, согласно известной формуле о решении задачи Дирихле для уравнения Пуассона (см. [2], [4] и др.), мы имеем

$$
u_{R G}\left(p_{0}\right)=\psi_{R}\left(p_{0}\right)+\int_{R G} Q_{R}\left(p_{0}, p_{1}\right) f\left(p_{1}\right) d V_{R}\left(p_{1}\right)
$$

где

$$
\psi_{R}\left(p_{0}\right)=\int_{\partial R G} K_{R}\left(p_{0}, p_{1}\right) \varphi_{R}\left(p_{1}\right) d S_{R}\left(p_{1}\right),
$$

$f=(L-\Delta) u_{R G}, Q_{R}\left(p_{1}, p_{2}\right)\left(p_{1}, p_{2} \in R G\right)$ - функция Грина для оператора Лапласа в области $R G$ и $K_{R}\left(p_{1}, p_{2}\right)$ - производная от функции Грина как функции от второго аргумента по направлению внутренней нормали к границе области, $d S_{R}(p), d V_{R}(p)$ - элементы поверхности и объема области $R G$. Таким образом, мы получаем интегральное уравнение относительно $u_{R G}$.

Сделаем замену переменных: $p_{i}=R \bar{p}_{i}\left(\bar{p}_{i} \in G, i=0,1\right)$. Пользуясь представлением

$$
Q_{R}\left(p_{1}, p_{2}\right)= \begin{cases}\frac{1}{(d-2) \omega_{d} r_{12}^{d-2}}+g\left(p_{1}, p_{2}\right), & d \geqslant 3 \\ \frac{1}{2 \pi} \ln \frac{1}{r_{12}}+g\left(p_{1}, p_{2}\right), & d=2\end{cases}
$$


и его единственностью (см. $\left[4\right.$, с. 302]), где $r_{i j}=\left|p_{i}-p_{j}\right|, \omega_{d}=$ $2 \pi^{(d / 2)} / \Gamma(d / 2)$ - площадь поверхности единичного шара в $d$-мерном пространстве, $g\left(p_{1}, p_{2}\right)$ - регулярная гармоническая функция, мы получаем соотношения

$$
\begin{aligned}
& R^{d-2} Q_{R}\left(R \bar{p}_{1}, R \bar{p}_{2}\right)=Q_{1}\left(\bar{p}_{1}, \bar{p}_{2}\right) \equiv Q\left(\bar{p}_{1}, \bar{p}_{2}\right), \\
& R^{d-1} K_{R}\left(R \bar{p}_{1}, R \bar{p}_{2}\right)=K_{1}\left(\bar{p}_{1}, \bar{p}_{2}\right) \equiv K\left(\bar{p}_{1}, \bar{p}_{2}\right) .
\end{aligned}
$$

Кроме того, если $F(R \bar{p})=\bar{F}(\bar{p})$ и $\bar{D}_{i}-$ дифференцирование по $i$-й координате точки $\bar{p}$, то $D_{i} F(R \bar{p})=\bar{D}_{i} \bar{F}(\bar{p}) / R$. Аналогично, $D_{i j} F(R \bar{p})=$ $\bar{D}_{i j} \bar{F}(\bar{p}) / R^{2}$. Отсюда

$$
\begin{aligned}
& \psi_{R}(R p) \equiv \int_{\partial R G} K_{R}\left(R p, p_{1}\right) \varphi_{R}\left(p_{1}\right) d S_{R}\left(p_{1}\right) \\
& =\int_{\partial G} K_{R}\left(R p, R p_{1}\right) \varphi_{R}\left(R p_{1}\right) R^{d-1} d S\left(p_{1}\right) \\
& =\int_{\partial G} K\left(p, p_{1}\right) \varphi\left(p_{1}\right) d S\left(p_{1}\right) \equiv \psi(p), \\
& \int_{R G} Q_{R}\left(R p, p_{1}\right)(L-\Delta) u_{R G}\left(p_{1}\right) d V_{R}\left(p_{1}\right) \\
& =\int_{G} Q_{R}\left(R p, R p_{1}\right)\left(\vec{L}_{R}-\frac{\Delta}{R^{2}}\right) \bar{u}_{R G}\left(p_{1}\right) R^{d} d V\left(p_{1}\right),
\end{aligned}
$$

где

$$
\bar{L}_{R} u=\sum_{i j} \bar{a}^{i j} \bar{D}_{i j}^{(1)} u / R^{2}+\sum_{i} \bar{b}^{i} \bar{D}_{i}^{(1)} u / R-\bar{c} u .
$$

По условию теоремы функция $\left(\bar{a}^{i j}-\delta^{i j}\right) / R$ ограничена в единичном шаре равномерно по всем $R>0$. Пользуясь этим, мы рассмотрим оператор $\tilde{L}_{R}=R \cdot\left(\bar{L}_{R}-\Delta / R^{2}\right)$, с помощью которого наше интегральное уравнение перепишем в виде

$$
\bar{u}_{R G}\left(p_{0}\right)=\psi\left(p_{0}\right)+R \int_{G} Q\left(p_{0}, p_{1}\right) \tilde{L}_{R}^{(1)} \bar{u}\left(p_{1}\right) d V\left(p_{1}\right) \quad\left(p_{0} \in G\right) .
$$

Отсюда

$$
\begin{aligned}
\bar{u}_{R G}\left(p_{0}\right)= & \psi\left(p_{0}\right)+R \int_{G} Q\left(p_{0}, p_{1}\right) \tilde{L}_{R}^{(1)} \psi\left(p_{1}\right) d V\left(p_{1}\right) \\
& +R^{2} \int_{G} Q\left(p_{0}, p_{1}\right) \tilde{L}_{R}^{(1)} \int_{G} Q\left(p_{1}, p_{2}\right) \tilde{L}_{R}^{(2)} \psi\left(p_{2}\right) d V\left(p_{2}\right) d V\left(p_{1}\right) \\
& +R^{3} \int_{G} Q\left(p_{0}, p_{1}\right) \tilde{L}_{R}^{(1)} \int_{G} Q\left(p_{1}, p_{2}\right) \tilde{L}_{R}^{(2)} \int_{G} Q\left(p_{2}, p_{3}\right) \\
& \times \tilde{L}_{R}^{(3)} \bar{u}_{R G}\left(p_{3}\right) d V\left(p_{3}\right) d V\left(p_{2}\right) d V\left(p_{1}\right) .
\end{aligned}
$$


Из этой формулы следует наше асимптотическое разложение. Чтобы это увидеть, запишем оператор $\tilde{L}_{R}$ в виде $\tilde{L}_{R} u=M u+R \cdot N u+\varepsilon u$, где

$$
\begin{aligned}
M^{(k)} u= & \sum_{i j} \sum_{m} a_{m}^{i j}(0) \pi_{m}\left(p_{k}\right) \bar{D}_{i j}^{(k)} u+\sum_{i} b^{i}(0) \bar{D}_{i}^{(k)} u \\
N^{(k)} u= & \frac{1}{2} \sum_{i j} \sum_{m n} a_{m n}^{i j}(0) \pi_{m}\left(p_{k}\right) \pi_{n}\left(p_{k}\right) \bar{D}_{i j}^{(k)} u \\
& +\sum_{i} \sum_{m} b_{m}^{i}(0) \pi_{m}\left(p_{k}\right) \bar{D}_{i}^{(k)} u-c(0) u \\
\varepsilon^{(k)} u= & \sum_{i j}\left(\frac{a^{i j}\left(R p_{k}\right)-\delta^{i j}}{R}-\sum_{m} a_{m}^{i j}(0) \pi_{m}\left(p_{k}\right)\right. \\
& \left.\quad-\frac{1}{2} R \sum_{m n} a_{m n}^{i j}(0) \pi_{m}\left(p_{k}\right) \pi_{n}\left(p_{k}\right)\right) \bar{D}_{i j}^{(k)} u \\
& +\sum_{i}\left(b^{i}\left(R p_{k}\right)-b^{i}(0)-R \sum_{m} b_{m}^{i}(0) \pi_{m}\left(p_{k}\right)\right) \bar{D}_{i}^{(k)} u \\
& -R \cdot\left(c\left(R p_{k}\right)-c(0)\right) u .
\end{aligned}
$$

В последнем члене коэффициенты перед функцией $u$ и ее производными имеют порядок $o(R)$ при $R \rightarrow 0$. Части $M$ и $N$ оператора $\tilde{L}_{R}$, не зависящие от $R$, при подстановке этого представления в формулу (12) дают члены разложения первого и второго порядков в формуле (9), а также члены высшего порядка малости. Нам остается доказать, что часть $\varepsilon$, подставленная в (12), вместе с членом третьего порядка в формуле (12), имеет порядок $o\left(R^{2}\right)$ равномерно по всем непрерывным $\varphi:(0 \leqslant \varphi \leqslant 1)$, заданным на границе области $G$. Для этого мы используем оценки решения задачи Дирихле для эллиптического дифференциального уравнения в терминах его коэффициентов и значения на границе области, которые можно найти, например, в книге [2].

Заменяя переменные, мы получаем, что $u(p) \equiv u_{R G}(R p)$ является решением задачи Дирихле для уравнения

$$
\sum_{i j} \bar{a}^{i j} \bar{D}_{i j} u+R \sum_{i} \bar{b}^{i} \bar{D}_{i} u-R^{2} \bar{c} u=0
$$

на $G$ со значением $u=\varphi$ на $\partial G$. По [2, с. 91$]$

$$
\begin{aligned}
|u|_{2, \alpha ; G}^{*} \equiv & \sup _{p \in G}|u(p)|+\sup _{p \in G} d_{p}|D u(p)|+\sup _{p \in G} d_{p}^{2}\left|D^{2} u(p)\right| \\
& +\sup _{p_{1}, p_{2} \in G} d_{p_{1}, p_{2}}^{2+\alpha} \frac{\left|D^{2} u\left(p_{1}\right)-D^{2} u\left(p_{2}\right)\right|}{\left|p_{1}-p_{2}\right|^{\alpha}} \leqslant C \sup _{G}|u|,
\end{aligned}
$$

где $D u$ - градиент функции $u, D^{2} u-$ матрица вторых производных функции $u, d_{p}=\operatorname{dist}(p, \partial G), d_{p_{1}, p_{2}}=\min \left(d_{p_{1}}, d_{p_{2}}\right), C=C\left(d, \alpha, \Lambda_{1}, \Lambda_{2}\right)$, 
$\alpha-$ коэффициент Гёльдера, равный в нашем случае единице, $\Lambda_{1}, \Lambda_{2}-$ нижняя и верхняя границы множества собственных чисел семейства матриц $\left(a^{i j}(p)\right)_{p \in S}$ :

$$
\left(\forall p_{1} \in S\right)\left(\forall p \in \mathbf{R}^{d} \backslash\{0\}\right) \quad \Lambda_{1}|p|^{2} \leqslant \sum_{i j} a^{i j}\left(p_{1}\right) p^{i} p^{j} \leqslant \Lambda_{2}|p|^{2} .
$$

При этом $\sup _{G}|u|=\sup _{\partial G}|u|[2$, с. 40].

С другой стороны, функция $u \equiv \int_{G} Q(\cdot, p) f(p) d V(p)$ является в $G$ решением задачи Дирихле для уравнения Пуассона $\Delta u=f$ с нулевым граничным условием. В этом случае по [2, с. 66] справедлива оценка

$$
|u|_{2, \alpha ; G}^{*} \leqslant C_{1}\left(\sup _{G}|u|+|f|_{2, \alpha ; G}^{(2)}\right) \text {, }
$$

где $C_{1}=C_{1}(d, \alpha)$ и

$$
|f|_{2, \alpha ; G}^{(2)}=\sup _{p \in G} d_{p}^{2}|f(p)|+\sup _{p_{1}, p_{2} \in G} d_{p_{1}, p_{2}}^{2+\alpha} \frac{\left|f\left(p_{1}\right)-f\left(p_{2}\right)\right|}{\left|p_{1}-p_{2}\right|^{\alpha}} .
$$

Кроме того,

$$
\sup _{G}|u| \leqslant \sup _{\partial G}|u|+C_{2} \frac{\sup _{G}|f|}{\Lambda_{1}},
$$

где $0<C_{2} \leqslant \exp ((\beta+1) d)-1, \beta=\sup _{G}|b| / \Lambda_{1}, b(p)=\left(b^{1}(p), \ldots, b^{d}(p)\right)-$ вектор коэффициентов при первых производных в уравнении (3) (см. $[2$, c. 43$])$.

Следовательно, член третьего порядка в формуле (12) имеет порядок $R^{3}$ равномерно по всем непрерывным $\varphi(0 \leqslant \varphi \leqslant 1)$, заданным на границе области $G$.

В то же время $u \equiv \psi(p)$ является решением задачи Дирихле для уравнения $\Delta u=0$ на $G$ и $u=\varphi$ на $\partial G$. Из тех же оценок следует, что интегралы, содержащие оператор $\varepsilon$, имеют порядок $o\left(R^{2}\right)$ равномерно по всем непрерывным $\varphi(0 \leqslant \varphi \leqslant 1)$, заданным на границе области $G$.

Для доказательства теоремы осталось выразить $u_{R G}\left(R p_{0}\right)$ и $\psi$ в виде интегралов (формулы (10) и (11)) и переменить порядок интегрирования.

Утверждение об ортогональности единице дополнительных членов первого и второго порядка следует из свойства (см. [4])

$$
\left(\forall p_{1} \in G\right) \quad \int_{\partial G} K\left(p_{1}, p_{2}\right) d V\left(p_{2}\right)=1 .
$$

Ортогональность $X_{1}$ любой линейной функции на границе следует из свойства

$$
\left(\forall p_{1} \in G\right) \quad \int_{\partial G} K\left(p_{1}, p_{2}\right)\left(\pi_{k}\left(p_{2}\right)-\pi_{k}\left(p_{1}\right)\right) d V\left(p_{2}\right)=0,
$$

которое является прямым следствием формулы Грина (см. [2]-[4]). Теорема 1 доказана. 
3.2. Доказательство теоремы 2. Известно (см. [2]-[4] и др.), что для шара $B=\left\{p \in \mathbf{R}^{d}:|p|<1\right\}$ функция Грина $Q_{B}$ равна

$$
Q_{B}\left(p_{1}, p_{2}\right) \equiv Q\left(p_{1}, p_{2}\right)= \begin{cases}\frac{1}{(d-2) \omega_{d}}\left(\frac{1}{r_{12}^{d-2}}-\frac{1}{\bar{r}_{12}^{d-2}}\right), & d \geqslant 3 \\ \frac{1}{2 \pi}\left(\ln \frac{1}{r_{12}}-\ln \frac{1}{\bar{r}_{12}}\right), & d=2\end{cases}
$$

где $\bar{r}_{12}^{2}=\left(1-r_{1}^{2}\right)\left(1-r_{2}^{2}\right)+r_{12}^{2}$. При $G=B$ ядро $K$, называемое ядром Пуассона, равно

$$
K_{B}\left(p_{1}, p_{2}\right) \equiv K\left(p_{1}, p_{2}\right)=\frac{1-r_{1}^{2}}{\omega_{d} r_{12}^{d}}
$$

где $r_{i}=\left|p_{i}\right|, r_{i j}=\left|p_{i}-p_{j}\right|$. Поэтому в первом основном члене асимптотики $i$-й коэффициент равен

$$
\begin{aligned}
& \int_{B} Q\left(0, p_{1}\right) D_{i}^{(1)} K\left(p_{1}, p_{2}\right) d V\left(p_{1}\right) \\
& = \begin{cases}\int_{B} \frac{1}{(d-2) \omega_{d}}\left(\frac{1}{r_{1}^{d-2}}-1\right) D_{i}^{(1)} \frac{1-r_{1}^{2}}{\omega_{d} r_{12}^{d}} d V\left(p_{1}\right), & d \geqslant 3, \\
\int_{B} \frac{1}{2 \pi} \ln \frac{1}{r_{1}} D_{i}^{(1)} \frac{1-r_{1}^{2}}{2 \pi r_{12}^{2}} d V\left(p_{1}\right), & d=2 .\end{cases}
\end{aligned}
$$

Интегрируя по частям по $i$-й компоненте и учитывая, что $Q\left(\cdot, p_{1}\right)=0$ при $p_{1} \in \partial B$, получаем

$$
= \begin{cases}-\int_{B} \frac{1}{(d-2) \omega_{d}} D_{i}^{(1)}\left(\frac{1}{r_{1}^{d-2}}-1\right) \frac{1-r_{1}^{2}}{\omega_{d} r_{12}^{d}} d V\left(p_{1}\right), & d \geqslant 3, \\ -\int_{B} \frac{1}{2 \pi}\left(D_{i}^{(1)} \ln \frac{1}{r_{1}}\right) \frac{1-r_{1}^{2}}{2 \pi r_{12}^{2}} d V\left(p_{1}\right), & d=2 .\end{cases}
$$

Оба этих выражения равны (при соответствуюших $d \geqslant 2$ ) следующему выражению:

$$
\frac{1}{\omega_{d}^{2}} \int_{B} \frac{\pi_{i}\left(p_{1}\right)}{r_{1}^{d}} \frac{1-r_{1}^{2}}{r_{12}^{d}} d V\left(p_{1}\right)
$$

Вычисление интеграла (14) для $d=2$ не представляет труда. Для произвольной размерности $d \geqslant 3$ сделаем замену переменных: $p \mapsto \bar{p}=$ $\mathscr{A} p$, где $\mathscr{A}$ - ортогональное преобразование, задаваемое матрицей (обозначаемой той же буквой), причем точка $p_{2}$ при этом преобразовании переходит в точку $(1,0, \ldots, 0)$, где $\left|p_{2}\right|=1$. Пусть $\left(\Phi_{1}, \ldots, \Phi_{d-1}\right)$ - сферические координаты точки $p_{2}$. Тогда матрицей такого преобразования 
будет, например,

$$
\mathscr{A}=\left(\begin{array}{rrrrrr}
C_{1} & S_{1} C_{2} & S_{1} S_{2} C_{3} & \cdots & S_{1} S_{2} \cdots S_{d-2} C_{d-1} & S_{1} S_{2} \cdots S_{d-1} \\
-S_{1} & C_{1} C_{2} & C_{1} S_{2} C_{3} & \cdots & C_{1} S_{2} \cdots S_{d-2} C_{d-1} & C_{1} S_{2} \cdots S_{d-1} \\
0 & -S_{2} & C_{2} C_{3} & \cdots & C_{2} S_{3} \cdots S_{d-2} C_{d-1} & C_{2} S_{3} \cdots S_{d-1} \\
\vdots & \vdots & \vdots & \ddots & \vdots & \vdots \\
0 & 0 & 0 & \cdots & C_{d-2} C_{d-1} & C_{d-2} S_{d-1} \\
0 & 0 & 0 & \cdots & -S_{d-1} & C_{d-1}
\end{array}\right) .
$$

Здесь $C_{i}=\cos \Phi_{i}, S_{i}=\sin \Phi_{i}$. Это преобразование можно получить как результат $d-1$ последовательных элементарных поворотов: $\mathscr{A}=\mathscr{A}_{1} \cdots \mathscr{A}_{d-1}$, где

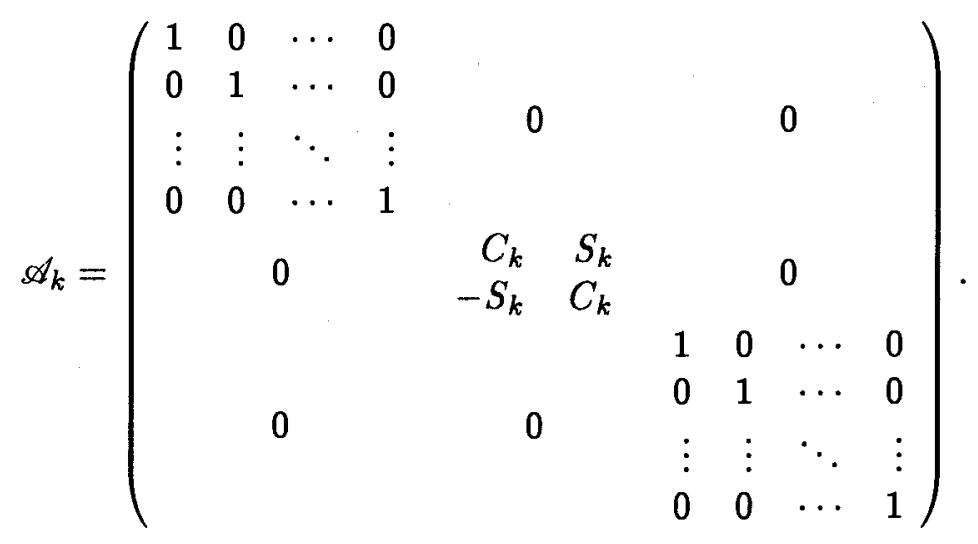

Имеем далее

$$
\frac{1}{\omega_{d}^{2}} \int_{B} \frac{\pi_{i}\left(p_{1}\right)\left(1-r_{1}^{2}\right)}{r_{1}^{d} r_{12}^{d}} d V\left(p_{1}\right)=\frac{1}{\omega_{d}^{2}} \int_{B} \frac{\pi_{i}\left(\mathscr{A}^{-1} \bar{p}_{1}\right)\left(1-r_{1}^{2}\right)}{r_{1}^{d} r_{12}^{d}} d V\left(\bar{p}_{1}\right)
$$

где во втором выражении $r_{12}^{2}=1+r_{1}^{2}-2 r_{1} c_{1}$ и $c_{1}-$ косинус угла между векторами $\bar{p}_{1}$ и $(1,0, \ldots, 0)$. Проще всего вычислить этот интеграл при первых номерах координат. Так, $\pi_{1}\left(\mathscr{A}^{-1} p\right)=C_{1} x-S_{1} y$, $\pi_{2}\left(\mathscr{A}^{-1} p\right)=S_{1} C_{2} x+C_{1} C_{2} y-S_{2} z$ и т.д., $k$-я координата представляется $k+1$ слагаемым. $\mathrm{K}$ счастью, в наших формулах общий случай можно свести к случаю первых номеров с помощью замены переменных. Пусть $T: \mathbf{R}^{d} \rightarrow \mathbf{R}^{d}$ - ортогональное преобразование, состоящее в перестановке координат: $T p=\left(p^{i_{1}}, \ldots, p^{i_{d}}\right)$, где $\left(i_{1}, \ldots, i_{d}\right)-$ перестановка чисел $(1,2, \ldots, d)$. Тогда

$$
\begin{aligned}
J\left(i_{1}, p_{2}\right) & \equiv \int_{B} \frac{\pi_{i_{1}}\left(p_{1}\right)}{F\left(\cos \left(\widehat{p_{1}, p_{2}}\right)\right)} d V\left(p_{1}\right)=\int_{B} \frac{\pi_{i_{1}}\left(T^{-1} \bar{p}_{1}\right)}{F\left(\cos \left(T^{-1} \bar{p}_{1}, p_{2}\right)\right)} d V\left(\bar{p}_{1}\right) \\
& =\int_{B} \frac{\pi_{1}\left(\bar{p}_{1}\right)}{F\left(\cos \left(\bar{p}_{1}, \widehat{T} p_{2}\right)\right)} d V\left(\bar{p}_{1}\right) \equiv J\left(1, T p_{2}\right)
\end{aligned}
$$


где $\left(\widehat{p}_{1}, p_{2}\right)-$ угол между векторами $p_{1}$ и $p_{2}$. Вычислим интеграл при $i=1$ :

$$
\begin{aligned}
& \frac{1}{\omega_{d}^{2}} \int_{B} \frac{x_{1}\left(1-r_{1}^{2}\right)}{r_{1}^{d} r_{12}^{d}} d V\left(p_{1}\right)=\frac{1}{\omega_{d}^{2}} \int_{B} \frac{\left(C_{1} x-S_{1} y\right)\left(1-r^{2}\right)}{r^{d} l^{d}} d V(p) \\
& =\frac{1}{\omega_{d}^{2}} \int_{0}^{1} \int_{0}^{2 \pi} \int_{0}^{\pi} \cdots \int_{0}^{\pi} \frac{C_{1} r c_{1}\left(1-r^{2}\right)}{r^{d} l^{d}} r^{d-1} s_{1}^{d-2} \cdots s_{d-2}^{1} d \varphi_{1} \cdots d \varphi_{d-1} d r \\
& =\frac{\omega_{d-1}}{\omega_{d}^{2}} \int_{0}^{1} \int_{0}^{\pi} \frac{C_{1} c_{1}\left(1-r^{2}\right)}{l^{d}} s_{1}^{d-2} d \varphi_{1} d r
\end{aligned}
$$

где $l^{2}=1+r^{2}-2 r c_{1},\left(r, \varphi_{1}, \ldots, \varphi_{d-1}\right)$ - полярные координаты точки $p$ и $c_{i}=\cos \varphi_{i}, s_{i}=\sin \varphi_{i}$.

Этот интеграл и ему подобные можно вычислять на основании равенства

$$
\begin{aligned}
1 & =\int_{B} K\left(p_{1}, p_{2}\right) d S\left(p_{2}\right)=\int_{0}^{2 \pi} \int_{0}^{\pi} \cdots \int_{0}^{\pi} \frac{1-r^{2}}{\omega_{d} l^{d}} s_{1}^{d-2} \cdots s_{d-2}^{1} d \varphi_{1} \cdots d \varphi_{d-1} \\
& =\omega_{d-1} \int_{0}^{\pi} \frac{1-r^{2}}{\omega_{d} l^{d}} s_{1}^{d-2} d \varphi_{1},
\end{aligned}
$$

откуда

$$
\int_{0}^{\pi} \frac{s_{1}^{d-2}}{l^{d}} d \varphi_{1}=\frac{\omega_{d}}{\omega_{d-1}\left(1-r^{2}\right)} .
$$

Интегрируя по частям внутренний интеграл, и используя формулу (15), получаем первый коэффициент

$$
\begin{aligned}
& \frac{\omega_{d-1}}{\omega_{d}^{2}} \int_{0}^{1} \int_{0}^{\pi} \frac{C_{1} c_{1}\left(1-r^{2}\right)}{l^{d}} s_{1}^{d-2} d \varphi_{1} d r \\
& =\frac{C_{1} \omega_{d-1}}{\omega_{d}^{2}} \int_{0}^{1}\left(1-r^{2}\right) \int_{0}^{\pi} \frac{c_{1} s_{1}^{d-2}}{l^{d}} d \varphi_{1} d r \\
& =\frac{C_{1} \omega_{d-1}}{\omega_{d}^{2}} \int_{0}^{1}\left(1-r^{2}\right) \frac{r \omega_{d}}{\omega_{d-1}\left(1-r^{2}\right)} d r=\frac{C_{1}}{2 \omega_{d}}
\end{aligned}
$$

Последнее выражение можно также переписать в виде $x_{2} /\left(2 \omega_{d}\right)$. Итак, в данном случае $J\left(1, p_{2}\right)=\pi_{1}\left(p_{2}\right) /\left(2 \omega_{d}\right)$. Для произвольного $k \in\{1, \ldots, d\}$ выберем перестановочный оператор $T$ таким образом, что $k=i_{1}$. При этом $J\left(k, p_{2}\right) \equiv J\left(i_{1}, p_{2}\right)=J\left(1, T p_{2}\right)=\pi_{1}\left(T p_{2}\right) /\left(2 \omega_{d}\right)=\pi_{k}(p) /\left(2 \omega_{d}\right)$. Отсюда коэффициент главного члена первого порядка равен

$$
\frac{1}{2 \omega_{d}} \sum_{i} b^{i}(0) \pi_{i}(p)
$$

Вычислим теперь коэффициент главного члена второго порядка:

$$
\int_{B} Q\left(0, p_{1}\right) K\left(p_{1}, p_{2}\right) d V\left(p_{1}\right)=\int_{B} \frac{1}{(d-2) \omega_{d}}\left(\frac{1}{r_{1}^{d-2}}-1\right) \frac{1-r_{1}^{2}}{\omega_{d} r_{12}^{d}} d V\left(p_{1}\right)
$$




$$
\begin{aligned}
& =\frac{\omega_{d-1}}{(d-2) \omega_{d}^{2}} \int_{0}^{1}\left(\frac{1}{r^{d-2}}-1\right)\left(1-r^{2}\right) r^{d-1} \int_{0}^{\pi} \frac{s_{1}^{d-2}}{l^{d}} d \varphi_{1} d r \\
& =\frac{\omega_{d-1}}{(d-2) \omega_{d}^{2}} \int_{0}^{1}\left(1-r^{2}\right)\left(1-r^{d-2}\right) r \cdot \frac{\omega_{d}}{\omega_{d-1}\left(1-r^{2}\right)} d r \\
& =\frac{1}{(d-2) \omega_{d}} \int_{0}^{1}\left(1-r^{d-2}\right) r \cdot d r=\frac{1}{2 d \omega_{d}}, \quad d \geqslant 3 .
\end{aligned}
$$

Та же формула справедлива для $d=2$ несмотря на другой вывод, который мы опускаем ввиду отсутствия каких-либо технических сложностей.

3.2.1. Вычисление ортогональных членов. Покажем на примере $d \geqslant 3$, как может быть вычислен ортогональный член $X_{1}$ коэффициента первого порядка. Имеем

$$
\begin{aligned}
& \int_{\partial B} X_{1}\left(0, p_{2}\right) \varphi\left(p_{2}\right) d S\left(p_{2}\right) \\
& \quad=\sum_{i j} \sum_{m} a_{m}^{i j}(0) \int_{B} Q\left(0, p_{1}\right) \pi_{m}\left(p_{1}\right) D_{i j}^{(1)} \psi\left(p_{1}\right) d V\left(p_{1}\right) .
\end{aligned}
$$

Здесь интеграл равен

$$
\begin{aligned}
& \int_{B} \frac{1}{(d-2) \omega_{d}}\left(\frac{1}{r_{1}^{d-2}}-1\right) \pi_{m}\left(p_{1}\right) D_{i j}^{(1)} \psi\left(p_{1}\right) d V\left(p_{1}\right) \\
& \quad=-\int_{B} \frac{1}{(d-2) \omega_{d}} D_{i}^{(1)}\left(\left(\frac{1}{r_{1}^{d-2}}-1\right) \pi_{m}\left(p_{1}\right)\right) D_{j}^{(1)} \psi\left(p_{1}\right) d V\left(p_{1}\right) .
\end{aligned}
$$

Принимаем, как и ранее, $m=1$ и $x_{1}=\pi_{1}\left(p_{1}\right)$. Дальнейшие формулы различаются для случаев возможного равенства индексов $i, j, 1$. Всего 5 вариантов: 1) $i \neq j, 1 \neq j, 1 \neq i, 2) i \neq j, 1=i, 3) i \neq j, 1=j$, 4) $i=j, 1 \neq i, 5) i=j=1$.

В первом случае имеем

$$
\begin{gathered}
-\int_{B} \frac{1}{(d-2) \omega_{d}} D_{i}^{(1)}\left(\left(\frac{1}{r_{1}^{d-2}}-1\right) x_{1}\right) D_{j}^{(1)} \psi\left(p_{1}\right) d V\left(p_{1}\right) \\
=\int_{B} \frac{x_{1}}{\omega_{d}} \frac{\pi_{i}\left(p_{1}\right)}{r_{1}^{d}} D_{j}^{(1)} \psi\left(p_{1}\right) d V\left(p_{1}\right) .
\end{gathered}
$$

Здесь мы снова прибегаем к интегрированию по частям, но краевые значения первой функции здесь не равны нулю:

$$
=\int_{B^{j}}\left[\frac{x_{1}}{\omega_{d}} \frac{\pi_{i}\left(p_{1}\right)}{r_{1}^{d}} \psi\left(p_{1}\right)\right]_{j_{a}}^{j_{b}} d V^{j}\left(p_{1}^{(j)}\right)-\int_{B} D_{j}^{(1)}\left(\frac{x_{1}}{\omega_{d}} \frac{\pi_{i}\left(p_{1}\right)}{r_{1}^{d}}\right) \cdot \psi\left(p_{1}\right) d V\left(p_{1}\right),
$$

где интегрирование по $d$-мерному шару $B$ мы заменили на последовательное интегрирование: наружное - по $(d-1)$-мерному шару $B^{j}$ (с изъятием $j$-й координаты) с переменной интегрирования $p_{1}^{(j)}$ и с элементом 
объема $d V^{j}\left(p_{1}^{(j)}\right)$, внутреннее - по $j$-й координате по интервалу $\left(j_{a}, j_{b}\right)$, где $-j_{a}=j_{b}=\sqrt{1-\left|p_{1}^{(j)}\right|^{2}}$. Замечаем, что на границе $B$ функция $\psi=\varphi$, а также, что $r_{1}=1$ и $j_{b}=j_{1}^{+} \equiv \pi_{j}\left(p_{1}^{+}\right)-$значение $j$-й координаты $p_{1}$, когда она находится на «верхней» границе, и $j_{a}=j_{2}^{-} \equiv \pi_{j}\left(p_{1}^{-}\right)-$значение $j$-й координаты $p_{1}$, когда она находится на «нижней» границе. Итак,

$$
\begin{aligned}
& \int_{B^{j}}\left[\frac{x_{1}}{\omega_{d}} \frac{\pi_{i}\left(p_{1}\right)}{r_{1}^{d}} \psi\left(p_{1}\right)\right]_{j_{a}}^{j_{b}} d V^{j}\left(p_{1}^{(j)}\right) \\
& =\int_{B^{j}} \frac{x_{1} \pi_{i}\left(p_{1}\right)}{\omega_{d} r_{1}^{d}}\left(\varphi\left(p_{1}^{+}\right)-\varphi\left(p_{1}^{-}\right)\right) d V^{j}\left(p_{1}^{(j)}\right) .
\end{aligned}
$$

Чтобы упростить этот интеграл, рассмотрим интеграл по поверхности шара

$$
\begin{aligned}
\int_{\partial B} F\left(p_{1}\right) d S\left(p_{1}\right) & =\int_{B^{j}}\left(F\left(p_{1}^{+}\right)+F\left(p_{1}^{-}\right)\right) \frac{1}{\sqrt{1-\left|p_{1}^{(j)}\right|^{2}}} d V^{j}\left(p_{1}^{(j)}\right) \\
& =\int_{B^{j}}\left(\frac{F\left(p_{1}^{+}\right)}{j_{1}^{+}}-\frac{F\left(p_{1}^{-}\right)}{j_{1}^{-}}\right) d V^{j}\left(p_{1}^{(j)}\right) .
\end{aligned}
$$

Мы замечаем, что этот интеграл равен предыдущему при

$$
F\left(p_{1}\right)=\frac{x_{1} \pi_{i}\left(p_{1}\right) \pi_{j}\left(p_{1}\right)}{\omega_{d}} \varphi\left(p_{1}\right) .
$$

Второй интеграл равен

$$
\int_{B}(-1) \frac{x_{1} \pi_{i}\left(p_{1}\right) \pi_{j}\left(p_{1}\right) d}{\omega_{d} r_{1}^{d+2}} \psi\left(p_{1}\right) d V\left(p_{1}\right)
$$

Помня о возможностях перестановочного оператора, рассмотрим этот интеграл для первых трех координат точки $p_{1}$ (для простоты изложения предположим, что $d \geqslant 5$ ):

$$
\begin{aligned}
& \int_{B}(-1) \frac{x_{1} y_{1} z_{1} d}{\omega_{d} r_{1}^{d+2}} \psi\left(p_{1}\right) d V\left(p_{1}\right) \\
& \quad=\int_{\partial B} \varphi\left(p_{2}\right)\left(\int_{B}(-1) \frac{x_{1} y_{1} z_{1} d}{\omega_{d} r_{1}^{d+2}} \frac{1-r_{1}^{2}}{\omega_{d} r_{12}^{d}} d V\left(p_{1}\right)\right) d S\left(p_{2}\right) .
\end{aligned}
$$

Внутренний интеграл равен

$$
\begin{aligned}
& -\frac{d}{\omega_{d}^{2}} \int_{B}\left(C_{1} x-S_{1} y\right)\left(S_{1} C_{2} x+C_{1} C_{2} y-S_{2} z\right) \\
& \quad \times\left(S_{1} S_{2} C_{3} x+C_{1} S_{2} C_{3} y+C_{2} C_{3} z-S_{3} w\right) \frac{1-r^{2}}{r^{d+2} l^{d}} d V(p),
\end{aligned}
$$


где $p=(x, y, z, w, \ldots), l^{2}=1+r^{2}-2 r c_{1}, c_{1}-$ косинус угла между $p$ и $(1,0, \ldots, 0)$. Последнее выражение равно

$$
\begin{array}{r}
-\frac{\omega_{d-3} d}{\omega_{d}^{2}} \int_{0}^{1} \int_{0}^{\pi} \int_{0}^{\pi} \int_{0}^{\pi}\left(1-r^{2}\right)\left(C_{1} S_{1}^{2} C_{2} S_{2} C_{3} x^{3}+C_{1}\left(C_{1}^{2}-2 S_{1}^{2}\right) C_{2} S_{2} C_{3} x y^{2}\right. \\
\left.-C_{1} C_{2} S_{2} C_{3} x z^{2}\right) \frac{1}{l^{d}} s_{1}^{d-2} s_{2}^{d-3} s_{3}^{d-4} d \varphi_{1} d \varphi_{2} d \varphi_{3} d r .
\end{array}
$$

Используя описанный выше метод вычисления интегралов (формула (15)), имеющих в знаменателе подынтегрального выражения $l^{d}$, получаем, что этот интеграл равен $(d-2) \omega_{d} x_{2} y_{2} z_{2} / 4$ (подробности мы опускаем за недостатком места). Вместе с первым членом это составляет $\pi_{i}\left(p_{2}\right) \pi_{j}\left(p_{2}\right) x_{2}(d+2) / 4$. Итак, в случае 1$)$ мы получаем слагаемое суммы, составляющей член $X_{1}: a_{1}^{i j} \pi_{i}\left(p_{2}\right) \pi_{j}\left(p_{2}\right) x_{2}(d+2) / 4\left(p_{2} \in \partial B\right)$. Аналогично вычисляются значения остальных четырех типов слагаемых суммы, составляющих член $X_{1}$.

Тот же метод вычисления применим к члену $X_{2}$. Мы вычислим ту компоненту этого члена, которая сохраняется в случае однородного в пространстве процесса, когда коэффициенты представляющего его дифференциального уравнения постоянны. В этом случае

$$
\begin{aligned}
X_{2}(0, p)=\sum_{i} b^{i} \sum_{k} b^{k} & \int_{B} Q\left(0, p_{1}\right) \\
& \times D_{i}^{(1)} \int_{B} Q\left(p_{1}, p_{2}\right) D_{k}^{(2)} K\left(p_{2}, p\right) d V\left(p_{2}\right) d V\left(p_{1}\right) .
\end{aligned}
$$

Имеем

$$
\begin{array}{rl}
\int_{B} & Q\left(0, p_{1}\right) D_{i}^{(1)} \int_{B} Q\left(p_{1}, p_{2}\right) D_{k}^{(2)} K\left(p_{2}, p\right) d V\left(p_{2}\right) d V\left(p_{1}\right) \\
& =-\int_{B} D_{i}^{(1)} Q\left(0, p_{1}\right) \int_{B} Q\left(p_{1}, p_{2}\right) D_{k}^{(2)} K\left(p_{2}, p\right) d V\left(p_{2}\right) d V\left(p_{1}\right) \\
& =\int_{B} D_{k}^{(2)} K\left(p_{2}, p\right) \int_{B} \frac{\pi_{i}\left(p_{1}\right)}{\omega_{d} r_{1}^{d}} Q\left(p_{1}, p_{2}\right) d V\left(p_{1}\right) d V\left(p_{2}\right)
\end{array}
$$

Вычислим внутренний интеграл

$$
\left\{\begin{array}{l}
\int_{B} \frac{\pi_{i}\left(p_{1}\right)}{\omega_{d} r_{1}^{d}} \frac{1}{(d-2) \omega_{d}}\left(\frac{1}{r_{12}^{d-2}}-\frac{1}{\bar{r}_{12}^{d-2}}\right) d V\left(p_{1}\right), d \geqslant 3, \\
\int_{B} \frac{\pi_{i}\left(p_{1}\right)}{2 \pi r_{1}^{2}} \frac{1}{2 \pi}\left(\ln \frac{1}{r_{12}}-\ln \frac{1}{\bar{r}_{12}}\right) d V\left(p_{1}\right), \quad d=2 .
\end{array}\right.
$$

С учетом перестановки координат заменяем $\pi_{i}$ на $x$, далее - замена переменных с помощью ортогонального преобразования и интегрирование по частям:

$$
\begin{aligned}
= & \frac{C_{1} \omega_{d-1}}{\omega_{d}^{2}(d-2)}\left(\int_{0}^{r_{2}} \int_{0}^{\pi} \frac{c_{1} s_{1}^{d-2}}{r_{2}^{d-2} l^{d-2}\left(r / r_{2}\right)} d \varphi_{1} d r\right. \\
& \left.+\int_{r_{2}}^{1} \int_{0}^{\pi} \frac{c_{1} s_{1}^{d-2}}{r^{d-2} l^{d-2}\left(r_{2} / r\right)} d \varphi_{1} d r-\int_{0}^{1} \int_{0}^{\pi} \frac{c_{1} s_{1}^{d-2}}{l^{d-2}\left(r r_{2}\right)} d \varphi_{1} d r\right) \quad(d \geqslant 3),
\end{aligned}
$$


где $l(r)=1+r^{2}-2 r c_{1}$. Для $d=2$ получаем

$$
\begin{aligned}
& \frac{C_{1}}{(2 \pi)^{2}} \int_{0}^{1} \int_{0}^{2 \pi} c_{1}\left(\ln \frac{1}{r_{12}}-\ln \frac{1}{\bar{r}_{12}}\right) d \varphi_{1} d r_{1} \\
& =\frac{C_{1}}{(2 \pi)^{2}} \int_{0}^{1} \int_{0}^{2 \pi} s_{1}^{2} r_{1} r_{2}\left(\frac{1}{r_{12}^{2}}-\frac{1}{\bar{r}_{12}^{2}}\right) d \varphi_{1} d r_{1} \\
& =\frac{C_{1}}{(2 \pi)^{2}}\left(\int_{0}^{r_{2}} \frac{r_{1}}{r_{2}} \int_{0}^{2 \pi} \frac{s_{1}^{2}}{l\left(r_{1} / r_{2}\right)} d \varphi_{1} d r_{1}+\int_{r_{2}}^{1} \frac{r_{2}}{r_{1}} \int_{0}^{2 \pi} \frac{s_{1}^{2}}{l\left(r_{2} / r_{1}\right)} d \varphi_{1} d r_{1}\right. \\
& \left.\quad-\int_{0}^{1} r_{1} r_{2} \int_{0}^{2 \pi} \frac{s_{1}^{2}}{l\left(r_{1} r_{2}\right)} d \varphi_{1} d r_{1}\right)
\end{aligned}
$$

После интегрирования с использованием формулы (15) получаем, что внутренний интеграл равен

$$
\begin{cases}\frac{C_{1} r_{2}}{2(d-2)^{2} \omega_{d}}\left(\frac{1}{r_{2}^{d-2}}-1\right), & d \geqslant 3 \\ \frac{C_{1} r_{2}}{2 \cdot 2 \pi} \ln \frac{1}{r_{2}}, & d=2\end{cases}
$$

где, согласно обозначению, $C_{1} r_{2}=x_{2}$. Возвращаемся к вычислению внешнего интеграла. Пользуясь равенством нулю внутреннего интеграла при $p_{2} \in \partial B$, получаем выражение

$$
\begin{cases}-\int_{B} \frac{1}{2(d-2)^{2} \omega_{d}^{2}} D_{k}^{(2)}\left[x_{2}\left(\frac{1}{r_{2}^{d-2}}-1\right)\right] \frac{1-r_{2}^{2}}{r_{23}^{d}} d V\left(p_{2}\right), d \geqslant 3, \\ -\int_{B} \frac{1}{2(2 \pi)^{2}} D_{k}^{(2)}\left[x_{2} \ln \frac{1}{r_{2}}\right] \frac{1-r_{2}^{2}}{r_{23}^{2}} d V\left(p_{2}\right), & d=2 .\end{cases}
$$

В случае $\pi_{k}\left(p_{2}\right) \neq x_{2}$ получаем для $d \geqslant 3$ :

$$
\begin{aligned}
& -\int_{B} \frac{x_{2}}{2(d-2) \omega_{d}}\left(-\frac{\pi_{k}\left(p_{2}\right)}{r_{2}^{d}}\right) \frac{1-r_{2}^{2}}{\omega_{d} r_{23}^{d}} d V\left(p_{2}\right) \\
= & \frac{1}{2(d-2) \omega_{d}^{2}} \int_{B} \frac{x_{2} \pi_{k}\left(p_{2}\right)\left(1-r_{2}^{2}\right)}{r_{2}^{d} r_{23}^{d}} d V\left(p_{2}\right) .
\end{aligned}
$$

Как и ранее, для простоты вычисления заменяем произвольную координату $\pi_{k}\left(p_{2}\right)$ на вторую, $y_{2}$; далее прибегаем к ортогональному преобразованию и интегрируем по частям; затем снова используем (15). В результате получаем, что последний интеграл равен $(d \geqslant 3)$

$$
\begin{aligned}
& \frac{x_{3} y_{3} \omega_{d-2}}{2(d-2) \omega_{d}^{2}} \int_{0}^{1} r\left(1-r^{2}\right) \int_{0}^{\pi} \int_{0}^{\pi} \frac{\left(c_{1}^{2}-s_{1}^{2} c_{2}^{2}\right) s_{1}^{d-2} s_{2}^{d-3}}{l^{d}} d \varphi_{1} d \varphi_{2} d r \\
& \quad=\frac{x_{3} y_{3}}{8(d-2) \omega_{d}}
\end{aligned}
$$

Для $d=2$ аналогичное выражение равно $(8 \cdot 2 \pi)^{-1} x_{3} y_{3}$.

В случае $\pi_{k}\left(p_{2}\right)=x_{2}$ получаем для $d \geqslant 3$ :

$$
-\int_{B} \frac{1}{2(d-2)^{2} \omega_{d}^{2}}\left(\frac{1}{r_{2}^{d-2}}-1-\frac{x_{2}^{2}(d-2)}{r_{2}^{d}}\right) \frac{1-r_{2}^{2}}{r_{23}^{d}} d V\left(p_{2}\right)
$$




$$
\begin{aligned}
& =-\frac{1}{2(d-2)^{2} \omega_{d}^{2}}\left(\frac{\omega_{d}}{2 d}-C_{1}^{2} \omega_{d-1} \int_{0}^{1}\left(1-r^{2}\right) r \int_{0}^{\pi} \frac{c_{1}^{2} s_{1}^{d-2}}{l^{d}} d \varphi_{1} d r\right. \\
& \left.\quad-S_{1}^{2} \omega_{d-2} \int_{0}^{1}\left(1-r^{2}\right) r \int_{0}^{\pi} \int_{0}^{\pi} \frac{c_{2}^{2} s_{1}^{d} s_{2}^{d-3}}{l^{d}} d \varphi_{1} d \varphi_{2} d r\right) \\
& =\frac{1}{8(d-2) \omega_{d}}\left(x_{3}^{2}-\frac{1}{d}\right) .
\end{aligned}
$$

И аналогично для $d=2$ получаем $(8 \cdot 2 \pi)^{-1}\left(x_{3}^{2}-\frac{1}{2}\right)$.

Совершая обратную перестановку индексов, окончательно получаем, что в случае однородного дифференциального уравнения

$$
X_{2}(0, p)= \begin{cases}\frac{1}{8(d-2) \omega_{d}}\left(\sum_{i, j=1}^{d} b^{i} b^{j} \pi_{i}(p) \pi_{j}(p)-\frac{1}{d} \sum_{i=1}^{d}\left(b^{i}\right)^{2}\right), & d \geqslant 3, \\ \frac{1}{8 \cdot 2 \pi}\left(\sum_{i, j=1}^{2} b^{i} b^{j} \pi_{i}(p) \pi_{j}(p)-\frac{1}{2} \sum_{i=1}^{2}\left(b^{i}\right)^{2}\right), & d=2 .\end{cases}
$$

Вычисление $X_{2}$ в неоднородном случае довольно громоздко в связи с необходимостью учесть совпадение индексов.

3.2.2. Замечание. Из полученного асимптотического разложения для переходной производяшей функции диффузионного процесса следует, что в однородном случае коэффициенты $b^{i}$ не могут зависеть от параметра $\lambda$ преобразования Лапласа, так как по этому параметру переходная функция должна убывать, а коэффициент перед $b^{i}$ в члене первого порядка меняет знак. От $\lambda$ зависит только коэффициент $c$. Для марковского процесса $c=c(\lambda, p)=\lambda c_{0}(p)$, где $c_{0}$ - некоторая функция.

Автор благодарен рецензенту журнала, учет замечаний которого способствовал улучшению стиля и полноте изложения.

\section{СПИСОК ЛИТЕРАТУРЫ}

1. Дынкия Е. Б. Марковские процессы. М.: Физматгиз, 1963, 859 с.

2. Гилбаре Д., Трудиягер $H$. Эллиптические дифференциальные уравнения с частными производными второго порядка. М.: Наука, 1989, 463 с.

3. Михлия С. Г. Курс математической физики. М.: Наука, 1968, 575 с.

4. Соболев С.Л. Уравнения математической физики. М.: ТТЛ, 1954.

5. Харламов Б. П. Случайные процессы с полумарховскими потоками первых вхождений. - Записки науч. семин. ЛОМИ, 1974, т. 41, с. 139-164.

6. Харламов Б. П. Переходные функции непрерывного полумарковского процесса. - Записки науч. семин. ЛОМИ, 1983, т. 130, с. 190-205.

7. Boradin A.N., Salminen P. Handbook of Brownian Motion - Facts and Formulae. Basel: Birkhäuser, 1996, 462 p.

Поступила в редакцию 25.VII.1997 